\title{
The measurement stand with DAQ card for recording a bioelectric signal from human muscles
}

\author{
Zbigniew Krawiecki ${ }^{1, *}$, Arkadiusz Hulewicz ${ }^{1}$, and Krzysztof Dziarski ${ }^{1}$ \\ ${ }^{1}$ Poznan University of Technology, Institute of Electrical Engineering and Electronics, 3A Piotrowo St., 60-965 Poznan, Poland
}

\begin{abstract}
The measurement stand for recording a bioelectric signal from human muscles has been described in the paper. A DAQ card with a USB bus has been used in the measuring channel. The acquisition of the electromyography signal is carried out using the non-invasive method using gel electrodes. The bioelectric signal is amplified, filtered and processed in hardware and software. The tests of system operation in the measurement laboratory have been carried out. The presented results relate to the measurement of the electromyography signal from the forearm muscles of the upper limb of a human.
\end{abstract}

\section{Introduction}

Human's physical activities are the result of his neuromuscular system. The generated electric charge and the action potential are measurable. The action potential of muscles is one of the many human bioelectrical signals. Others are, for example: ECG (electrocardiogram), EEG (electroencephalogram), VEP (visual evoked potential), or biological signals like PPG (photoplethysmogram) [1-4]. The thermovision cameras are sometimes used to monitor biological objects. In this case, the attention should be paid to the parameters of the used converters, as described in $[5,6]$.

Bioelectric signals can be recorded in a non-invasive way from the human skin surface. Such signal is characterized by a relatively small amplitude from tens of $\mu \mathrm{V}$ to several $\mathrm{mV}$. The voltage of such a signal is not measured by typical digital multimeters (AC voltmeters) $[7,8]$. The measurement of such a signal requires the use of a sensitive input circuit that will amplify the bioelectric signal and at the same time reduce the impact of undesired signals [9-11].

A measurement stand with a DAQ card, which was made for the acquisition and processing of an electromyography signal (EMG) has been presented in this paper. It is expected to use this position in didactics. However, in the long-term, by extending advanced algorithms for the processing and analysis of bioelectric signals to scientific research [12-14].

\section{Measuring stand and the registration results}

\subsection{Assumptions}

In general, it was assumed that the measuring stand will be used for recording, processing and archiving EMG signal from human muscles. The measuring channel will be composed of an analogue part and a digital part. Surface electrodes, amplifying stage with a bandpass filter will form an analog part of the measurement stand. The digital part: DAQ card and computer with software. It was recognized that this concept of the measurement stand enables easy hardware and software development. These features can be important when testing the performance of new signal processing algorithms for medical diagnosis purposes. The possibility of a reliable assessment of muscles electrical activity has a particular importance for the effectiveness of rehabilitation procedures $[15,16]$.

It was assumed that the electromyography signal will be obtained using disposable, passive gel electrodes. Then, the analog signal will be amplified, it will be filtered and converted to a digital signal (NI USB 6211). Further signal processing will be performed programmatically and the results visualized on the computer screen. The obtained results will be archived on the computer disk in a text file. The components used in the measurement system will be powered with a low voltage safe for humans. The software and hardware resources of the computer will be used as much as possible.

\subsection{The implementation of the stand and the results of measurements of the EMG signal}

One of the most important elements of the measuring channel is the pre-amplifier. This pre-amplifier was built using the INA128. It is an instrumental amplifier that is often recommended for measuring bioelectric signals. In the feedback system, a cut-off filter for DC voltage component and low-frequency $\mathrm{AC}$ voltage component was used. The typical configuration of this amplifier has been modified with an additional feedback circuit. A socalled Right-Leg-Drive circuit (RLD) is used to measure 
the ECG signal. In the measurement system, this circuit improves the Common Mode Rejection Ratio (CMRR). The minimum catalog value of the $C_{M R R_{\text {INA128(dB) }} \text { for }}$ the amplification value of $10 \mathrm{~V} / \mathrm{V}$ is $93 \mathrm{~dB}$ (INA128PA, UA). The RLD circuit introduces additional $\mathrm{CMRR}_{\mathrm{RLD}(\mathrm{dB})}$ attenuation. The attenuation value determined during the simulation is $20 \mathrm{~dB}(50 \mathrm{~Hz})$. The resultant value of $\mathrm{CMRR}_{(\mathrm{dB})}$ attenuation is described by the formula (1).

$$
C M R R_{(\mathrm{dB})}=C M R R_{\mathrm{RLD}(\mathrm{dB})}+C M R R_{\mathrm{INA} 128(\mathrm{~dB})}
$$

The actual $\mathrm{CMRR}_{(\mathrm{dB})}$ of the pre-amplifier has not been determined. The research has been carried out in the frequency range from $1 \mathrm{~Hz}$ to $500 \mathrm{~Hz}$. The output signal of the amplifier was not measurable by the available equipment. In this situation, it was assumed that the obtained results are satisfactory. Before converting the analog EMG signal into a digital form, a low-pass filter with a $500 \mathrm{~Hz}$ cut-off frequency was used. The circuits: the pre-amplifier with a filter, a measuring card, a computer and a software create a stand with the functionality of a virtual instrument. The hardware part performs signal processing tasks such as conditioning, filtering, digital conversion and data visualization. The software part is: a system control, digital signal filtration, signal analysis, a data formatting for visualization and archiving. Figure 1 shows the functional construction of the measuring stand. The functional structure maps the main implemented tasks.

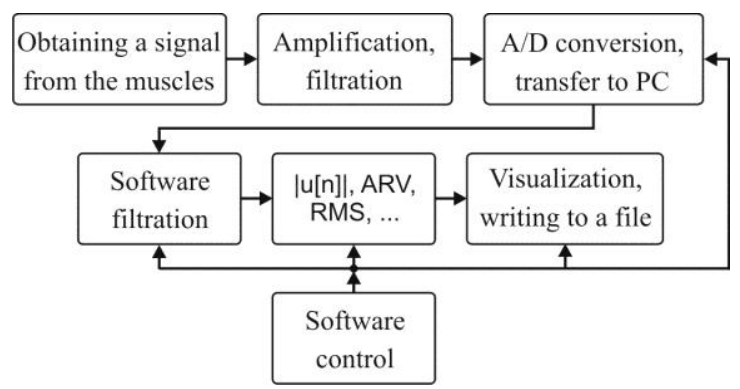

Fig. 1. The functional construction of the measuring stand.

A measuring stand with a DAQ card for EMG signal registration has been launched and tested in a measuring laboratory. After this stage, the electromyography signal was measured. The surface electrodes were put on the skin over the biceps of the right hand arm. The examples of the results are presented in Figure 2.

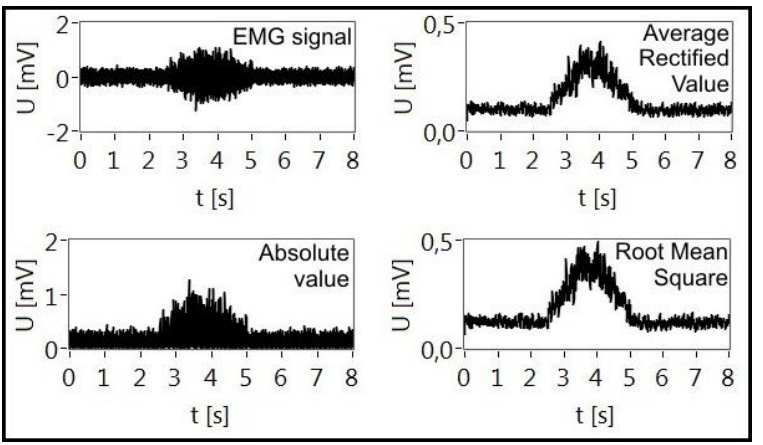

Fig. 2. The example results of measurement and processing of EMG signal.

\section{Summary}

The measuring amplifier with the RLD (Right-Leg Drive) circuit has an increased CMRR in relation to the amplifier specification. The configuration of the amplifier with the RLD circuit is designed to measure the ECG signal. The authors of the paper built a computer measuring stand with an amplification stage and an RLD circuit to check if such a solution would be applicable when measuring the EMG signal. The obtained result is positive, which was confirmed by laboratory measurements. Adopting the system intended for the measurement of the electrocardiographic signal for the purposes of testing electrical muscle activity, it is advisable to adjust the circuit's amplification and modify the bandpass filter. Considering the structure of the computer measurement stand, it is possible to carry out its relatively simple expansion with additional measuring channels, reproducing the blocks of input circuits. The limit is the number of DAQ card measurement channels.

\section{References}

1. R. Merletti, P.A. Parker, Electromyography: Physiology, Engineering, and Noninvasive Applications, (Wiley-IEEE Press, 2004)

2. C.J. De Luca, J.G. Webster, Encyclopedia of medical devices and instrumentation, (JWP, 2006)

3. A. Hulewicz, IJET, 65, 39 (2019)

4. D. Prokop, A. Cysewska-Sobusiak, A. Hulewicz, Proc. Eng. 47, 422 (2012)

5. K. Dziarski, J. Parzych,. ITM Web of Conf., 19, 01038 (2018)

6. J. Parzych, Z. Krawiecki, Przeg. Elektr., 10, 86-87, (2010)

7. P. Otomanski, Z. Krawiecki, A. Odon, JPCS, 238, 012005 (2010).

8. A. Odon, Z. Krawiecki, Meas., 44, 8, 1406-1411, (2011)

9. J.V. Basmajian, C.J. De Luca, Muscles Alive: their functions revealed by electromyography, (W\&W, Baltimore, Maryland, 1985)

10. C.J. De Luca, D.L. Gilmore, M. Kuznetsov, S.H. Roy, J. of Biom., 46, 1573-1579, (2010)

11. M.B.I. Reaz, M.S. Hussain, F. Mohd-Yasin, Biol. Proced. Online, 8(1), 11-35, (2006)

12. A. Lukyanchikov, A. Melnikov, O. Lukyanchikov, ITM Web of Conf. 18, 02001 (2018)

13. A. Merlo, D. Farina. IEEE Trans. Biomed. Eng. 50(3), 316-323, (2003)

14. B-K Zhang, G-L Zhong, H. Deng, ITM Web of Conf., 7, 09016 (2016)

15. M. Grzeskowiak, Z. Krawiecki, W. Labedz, J. Kaczmarczyk, J. Lewandowski, D. Lochynski, J. of Sport Rehab. (to be published)

16. D.K. Kumar, N.D. Pah, A. Bradley, IEEE Trans Neural. Syst. Rehab. Eng., 11(4):400-406, (2003) 